\title{
Update of human and mouse matrix metalloproteinase families
}

\author{
Brian C. Jackson,' Daniel W. Nebert ${ }^{2 *}$ and Vasilis Vasiliou ${ }^{*}$ \\ 'Molecular Toxicology and Environmental Health Sciences Program, Department of Pharmaceutical Sciences, University of Colorado \\ Denver, Aurora, CO 80045, USA \\ ${ }^{2}$ Department of Environmental Health and Center for Environmental Genetics (CEG), University of Cincinnati Medical Center, \\ Cincinnati, OH 45267-0056, USA \\ *Correspondence to: E-mail: dan.nebert@uc.edu; vasilis.vasiliou@ucdenver.edu
}

Date received (in revised form): 31st October, 2009

\begin{abstract}
Matrix metalloproteinases (MMPs) are a family of zinc proteases that degrade most of the components of the extracellular matrix (ECM). MMPs also have a number of non-traditional roles in processing factors related to cell growth/proliferation, inflammation and more. There are 23 human MMPs and 23 mouse MMPs, most of which share orthology among most vertebrates; other examples have been found in invertebrates and plants. MMPs are named in order of discovery, but also have been grouped by domain structure or by phylogenetic analysis. MMPs are multi-domain proteins which generally contain a signal sequence; propeptide (which keeps the protein inactive until cleaved); catalytic domain; and a hemopexin-like domain (which provides substrate specificity). MMPs are thought to play a role in many disease states, including arthritis, vascular disease, lung injury, wound repair, cancer and various neurodegenerative disorders. Although there has been much clinical interest in MMP inhibitors (MMPIs), few trials have been successful — often due to the broad nature of inhibition and the complex role of different MMPs in a given disease state.
\end{abstract}

Keywords: MMPs, MMPIs, zinc endopeptidases, gene family, evolution, disease, cell growth, proliferation, inflammation, arthritis, vascular disease, lung injury, wound repair, cancer, neurodegenerative disorders

\section{Introduction}

The extracellular matrix (ECM) plays a role in adhesion, growth, apoptosis and cell-cell communication, and regulation of these functions is critical to cellular activities. One family of proteins which functions in the regulation of the ECM is the matrix metalloproteinases (MMPs; also called matrixins). MMPs belong to the metzincin superfamily (zinc endopeptidases), which consists of ADAMs (a disintegrin and metalloproteinase), ADAMTSs (a disintegrin and metalloproteinase with thrombospondin motifs), bone morphogenetic protein 1/tolloid-like metalloproteinases (BMPI/ TLLs), meprins and MMPs. ${ }^{1}$ MMPs are zinc proteases which have the ability to degrade most components of the ECM, as well as act on an extremely broad array of extracellular components. ${ }^{2}$ The first MMP - an interstitial collagenase (MMP1) — was discovered by Gross et al. ${ }^{3}$ in 1962, and had the ability to degrade collagen. In the Matrix Metalloproteinase Conference (Destin, Florida 1989), MMPs were assigned sequential names and defined using biochemical characteristics, including: appearance in a latent form; inhibition by tissue inhibitor of metalloproteinase (TIMP); ability to hydrolyse at least one ECM component; and presence of zinc as an intrinsic metal ion. ${ }^{4}$ Since then, research on MMPs has expanded greatly, and 23 human and 23 mouse MMPs are known, of which most, but not all, share orthology.

MMPs are typically synthesised as preproenzymes and secreted immediately (exceptions 
include MMP8 and MMP9, which can be stored in the granules of neutrophils ${ }^{5}$ ) in an inactive form. Activation of MMPs can occur extracellularly by proteolytic cleavage by other MMPs or by serine proteinases (eg plasmin). Alternatively, MMPs with a furin recognition sequence (MT-MMPs, MMP11, MMP21, MMP23B and MMP28) are activated in the Golgi apparatus and released in a proteolytically active form. Active MMPs are regulated by inhibitors such as TIMPs and the general plasma proteinase inhibitor $\alpha_{2}$-macroglobulin. ${ }^{6}$ Other forms of regulation of MMPs include transcriptional control and control of translation and secretion. Expression of MMPs can be initiated by transcription factors, including activator protein-1 (AP-1), nuclear factor kappaB (NF-кB) and serum amyloid A-activating factor (SAF-1), especially via pro-inflammatory cytokines. ${ }^{7}$

MMPs are multi-domain proteins, which typically contain a signal sequence (targets the peptide for secretion); a propeptide; a catalytic domain; a linker domain (also called a hinge region); and a hemopexin-like domain. ${ }^{8}$ Figure 1 shows the domain composition of all of the human MMPs. The catalytic domain (metalloproteinase activity) contains a well-conserved zinc-binding motif (HEXXHXXGXXH), in which three histidines bind the catalytic zinc ion. In the inactive form, the cysteine switch motif (PRCGXPD; present in the propeptide domain of all human MMPs except
MMP23B) keeps pro-MMPs latent by a Cys- $\mathrm{Zn}^{2+}$ interaction, which prevents a key hydrolytic water from binding to the zinc ion; ${ }^{9}$ removal of parts of the propeptide and cysteine switch allows activation of the enzyme. The hemopexin-like domain contributes to substrate and/or ligand specificity, subcellular localisation and activation/inhibition of various MMPs. ${ }^{10}$ In gelatinases, the gelatin-binding domain (fibronectin type II motif) is also thought to play a role in substrate specificity.

In addition to their cellular matrix degrading activities, MMPs play a role in the proteolytic activation or degradation of many other molecules, especially those related to inflammation. ${ }^{7}$ MMPs are thought to affect leukocyte infiltration of tissues from the peripheral circulation - directly by degradation of the basement membrane by MMP2 and MMP9 and indirectly by regulating expression of adhesion molecules. MMP2 cleaves big endothelin-1 (ET-1) to a peptide that binds the endothelin A receptor, which upregulates integrins involved in leukocyte rolling arrest and subsequent trans-endothelial migration. ${ }^{11}$ MMP9 (and possibly MMP1, MMP2 and MMP3) have been shown to degrade $\alpha 1$-proteinase, inactivating its enzymatic activity but creating two fragments that remain bound to each other under physiological conditions. The $M_{r}=4,200$ cleaved $\alpha 1$-proteinase fragment is a chemoattractant for polymorphonuclear leukocytes. $^{12}$ MMPs have been shown to cleave

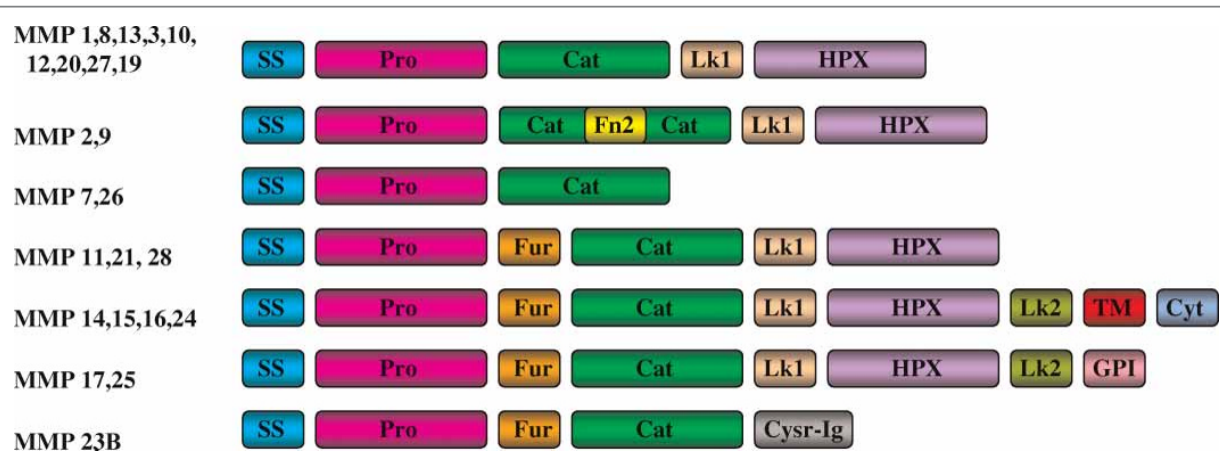

Figure I. Domain composition of human MMPs. Abbreviations: SS, signal sequence; Pro, propeptide; Fur, furin cleavage site; Cat, catalytic domain; Fn2, fibronectin type II motif; LkI, Linker domain type I; Hpx, hemopexin-like domain; Lk2, linker domain type 2; TM, transmembrane domain; GPI, glycosylphosphatidylinositol anchor; Cyt, cytoplasmic tail; Cysr-lg, cysteine rich and Ig-like domain (based on Nagase et al. ${ }^{8}$ and Cauwe et al. $^{2}$ ). 
pro-inflammatory cytokines, leading to their activation. MMP1, MMP7, MMP9, MMP14 and MMP17 have been shown to activate tumour necrosis factor (TNF). ${ }^{13,14}$ Interleukin-1 beta (IL- $\beta$ ) is activated by MMP1, MMP2, MMP3 and MMP9, ${ }^{15}$ and transforming growth factor-beta (TGF- $\beta$ ) is activated by MMP3, MMP7, MMP9 and MMP14. ${ }^{16,17}$ Cleavage of chemokines by MMPs can act to either induce or inhibit inflammation. ${ }^{7}$

\section{The MMP gene family}

There are 23 known human MMPs and 23 in the mouse. Human MMP protein sequences were retrieved from the HUGO Gene Nomenclature Committee site (http://www.genenames.org/), and mouse MMP protein sequences were retrieved from Mouse Genome Informatics (http://www. informatics.jax.org/). Table 1 lists all human and mouse MMPs, along with trivial names, group identification and chromosome location. In addition, there is one known human pseudogene, MMP23A (not listed). Figure 2 is a dendrogram of human MMPs, showing likely orthology. It is divided into four groups based on likely phylogenetic relationships. In addition to trivial names, MMPs traditionally have been designated by increasing numbers as new enzymes are discovered. Thus, collagenase, gelatinase and stromelysin were assigned as MMP1, MMP2 and MMP3, respectively. Some designations (eg MMP4, MMP5 and MMP6) are not used because each represents a gene product which was previously given a different MMP number. In most cases, the mouse enzyme is designated with the name of its human orthologue, with the following exceptions: human MMP1 has two shared orthologues - mouse MMP1a and MMP1b; human MMP26 has no clear mouse orthologue; and human MMP3 and MMP10 cluster more closely to each other than to mouse MMP3 and MMP10.

Also traditionally, MMPs are divided into six groups based on domain organisation and substrate preference: collagenases, gelatinases, stromelysins, matrilysins, membrane-type (MT) MMPs and others. ${ }^{8}$ The collagenases (MMP1, MMP8,
MMP13) were the first to be discovered and may be considered the prototypical MMPs. These enzymes can cleave interstitial collagens. MMP2 and MMP14 have the ability to cleave collagen as well, but are classified differently based on their domain arrangement. Collagenases contain the signal sequence; propeptide; catalytic domain with cysteine switch motif; linker region; and hemopexin-like domains. Gelatinases (MMP2, MMP9) have a similar domain composition to that of the collagenases, but in addition have three repeats of a fibronectin type II motif, which allows binding to gelatin/collagen and digestion of gelatin. The domain arrangement of stromelysins (MMP3, MMP10, MMP11) is similar to that of the collagenases, but lacks the ability to cleave interstitial collagens. Stromelysin 3 (MMP11) is unusual in that it contains a recognition sequence for furintype convertases and is thus activated intracellularly. MMP11 has limited activity towards ECM substrates. $^{18}$ Matrilysins (MMP7, MMP26) lack a hemopexin-like domain. The membrane-type MMPs can be divided into two groups: those which contain a type I transmembrane domain (MMP14, MMP15, MMP16, MMP24) and those which contain a glycosylphosphatidylinositol (GPI) anchor (MMP17 and MMP25). All membrane-type MMPs are activated intracellularly (they contain a furin cleavage site) and are active at the cell surface.

The remaining MMPs are put into an 'others' group. MMP12, MMP20 and MMP27 have structural and location similarities to stromelysins. MMP23B lacks a hemopexin-like domain (similar to matrilysins), which is replaced by a C-terminal cysteine-rich immunoglobulin-like domain. Furthermore, the propeptide of MMP23B is the only MMP that lacks a cysteine switch - the propeptide region has a transmembrane domain, but it is in the propeptide and the enzyme is released from the cell surface upon cleavage. In addition, MMP21, MMP23B and MMP28 have a furin cleavage site.

For more comprehensive lists of MMP substrates in vitro and in vivo, see the protease databases CutDB (http://cutdb.burnham.org) and MEROPS (http://merops.sanger.ac.uk) and the recent review of Cauwe et al. ${ }^{2}$ 
Table I. List of all human and mouse MMPs, with MMP number, trivial name, grouping by domain composition, grouping by phylogenetic alignment and chromosomal location.

\begin{tabular}{|c|c|c|c|c|c|c|}
\hline \multirow[b]{2}{*}{ MMP \# } & \multirow[b]{2}{*}{ Trivial name } & \multicolumn{2}{|c|}{ Group } & \multirow{2}{*}{$\begin{array}{l}\text { Human } \\
\text { Chromosome }\end{array}$} & \multicolumn{2}{|c|}{ Mouse } \\
\hline & & Domain & Phylogeny & & Orthologue & Chromosome \\
\hline MMPI & $\begin{array}{l}\text { Interstitial } \\
\text { collagenase }\end{array}$ & Collagenases & A & IIq21-q22 & MMPIa, Ib & 9 \\
\hline MMP8 & $\begin{array}{l}\text { Neutrophil } \\
\text { collagenase }\end{array}$ & Collagenases & A & $1|q 2|-q 22$ & MMP8 & 9 \\
\hline MMPI3 & Collagenase 3 & Collagenases & $A$ & I Iq22.3 & MMPI3 & 9 \\
\hline MMP2 & Gelatinase A & Gelatinases & $A$ & $16 q|3-q 2|$ & MMP2 & 8 \\
\hline MMP9 & Gelatinase B & Gelatinases & A & $20 q|2-q| 3$ & MMP9 & 2 \\
\hline MMP7 & Matrilysin I & Matrilysins & A & $1|q 2|-q 22$ & MMP7 & 9 \\
\hline MMP26 & Matrilysin 2 & Matrilysins & $A$ & IIpI5 & & \\
\hline MMP3 & Stromelysin I & Stromelysins & $A$ & I Iq22.3 & MMP3 & 9 \\
\hline MMPIO & Stromelysin 2 & Stromelysins & A & I Iq22.3 & MMPIO & 9 \\
\hline MMPII & Stromelysin 3 & Stromelysins & B & $22 q 11.23$ & MMPII & 10 \\
\hline MMPI4 & MTI-MMP & Membrane TM & B & $|4 q| 1-q \mid 2$ & MMPI4 & 14 \\
\hline MMPI5 & MT2-MMP & Membrane TM & B & $16 q 13$ & MMPI5 & 8 \\
\hline MMPI6 & MT3-MMP & Membrane TM & B & $8 q 21$ & MMPI6 & 4 \\
\hline MMP24 & MT5-MMP & Membrane TM & B & $20 q \mid 1.2$ & MMP24 & 2 \\
\hline MMPI7 & MT4-MMP & Membrane GPI & B & $12 q 24.3$ & MMPI7 & 5 \\
\hline MMP25 & MT6-MMP & Membrane GPI & B & $|6 p| 3.3$ & MMP25 & 17 \\
\hline MMPI 2 & $\begin{array}{l}\text { Macrophage } \\
\text { elastase }\end{array}$ & Other & $A$ & I Iq22.3 & MMPI2 & 9 \\
\hline MMP20 & Enamelysin & Other & $A$ & I Iq22.3 & MMP20 & 9 \\
\hline MMP27 & - & Other & A & IIq24 & MMP27 & 9 \\
\hline MMPI9 & - & Other & C & $12 q \mid 4$ & MMPI9 & 10 \\
\hline MMP2I & - & Other & C & $10 q 26.3$ & MMP2I & 7 \\
\hline MMP23B & CA-MMP & Other & D & Ip36.3 & MMP23B & 4 \\
\hline MMP28 & Epilysin & Other & C & $17 q 21.1$ & MMP28 & 11 \\
\hline
\end{tabular}

\section{Evolution of the MMPs}

To consider the evolutionary relationships between individual MMPs, a neighbour-joining dendrogram of human MMPs was constructed using the alignment tool at http://align.genome.jp/ and default parameters (Figure 2). There appear to be four major groups (labelled A, B, C and D in Figure 2): group A consists of the collagenases, stromelysins (less 


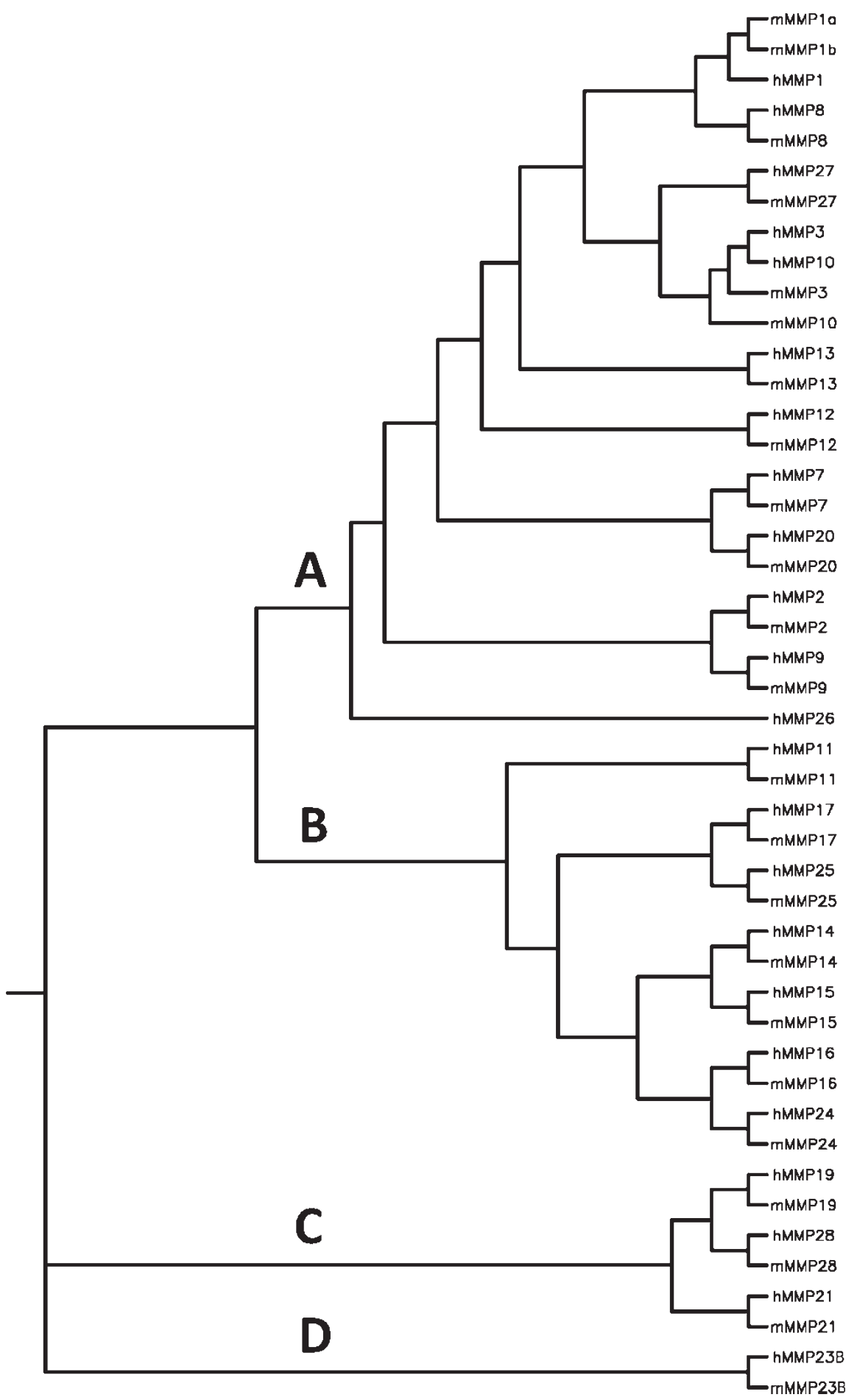

Figure 2. Dendrogram of human (hMMP) and mouse (mMMP) matrix metalloproteinases, showing likely orthology, and divided into four divergent groups, based on likely phylogenetic relationships.

stromelysin 3/MMP11), matrilysins and macrophage including both the GPI-anchored and transmemelastase/MMP12, enamelysin/MMP20, and MMP27; brane MMPs, as well as stromelysin 3; group C congroup B includes all of the membrane-bound MMPs, sists of the ungrouped members MMP19, MMP21 
and epilysin/MMP28; and group D consists of MMP23B alone.

From our current analysis, a close relationship between individual mouse and human MMPs implies that diversification of MMPs occurred before the two groups diverged 65-70 million years ago and that human and murine MMPs evolved in parallel. Similar probable orthology has been found in various vertebrates - including human, rat, mouse, rabbit and chicken. In addition to those found in vertebrates, MMPs have been found in invertebrates and plants, although these tend to have much simpler domain structures. Furthermore, Bacteroides fragilis metalloproteinase toxin-2 has sequence similarity to the conserved regions of MMPs, indicating that the origin of MMPs may be traceable back to bacteria. ${ }^{19}$ Massova et al. ${ }^{19}$ also found similar groupings in evolutionary analyses of the entire amino acid sequence and the hemopexin-like and catalytic domains, indicating that domain assemblage was an early evolutionary event. In at least one example (MMP7), it is likely that a domain was truncated.

\section{MMPs in disease}

It is well known that the ECM plays a key role in tissue structure and function, so it is not surprising that MMPs have been implicated in the pathogenesis and progression of many diseases - including arthritis, vascular disease, lung injury, cancer and some neurodegenerative disorders. ${ }^{20}$ MMPs are especially likely to play a role in cases where key events involve matrix degradation and/or inflammation. Uncontrolled MMP activity in rheumatoid arthritis (RA) damages joints by degrading cartilage and bone, as well as by stimulating angiogenesis and inflammation. MMP1, MMP2, MMP3, MMP9, MMP13 and MMP14 are elevated in the serum and joints of patients with RA, and MMP9 levels (from macrophages and neutrophils) have been correlated with RA severity. ${ }^{21}$ In an antibody-induced model of arthritis, Mmp9(-/-) knockout mice show milder arthritis than that in control mice; however, Mmp2(-/-) knockout mice display a much more severe clinical and histological arthritis compared with that in wild-type controls. ${ }^{22}$

MMPs play a role in many aspects of wound healing, including degradation of the ECM, induction of inflammation and recruitment of cells for re-epithelialisation and angiogenesis. ${ }^{23}$ Although it would often be advantageous to limit the inflammatory phase of wound repair, long-term broadspectrum MMP inhibition often leads to slower wound healing overall.

Among changes to the vasculature, MMP activity has been linked to aneurysm expansion and rupture, events in most all stages of development of atherosclerotic plaque and myocardial infarction, likelihood of cardiac rupture and disruption of the blood-brain barrier during stroke. ${ }^{20}$

Among lung injuries, MMPs have been implicated in acute respiratory distress syndrome (ARDS) and chronic obstructive pulmonary disease (COPD), which encompasses both emphysema and chronic bronchitis. ${ }^{24}$ MMP1, MMP8, MMP9 and MMP12 have been implicated in COPD disease progression. $^{25}$ MMPs contribute to destruction of elastic tissues and fibrosis, as well as recruitment and effects of inflammatory cells. As one example, Mmp12(-/-) (macrophage elastase) knockout mice fail to develop emphysema after six months of exposure to cigarette smoke, whereas wild-type mice are susceptible. ${ }^{26}$

There has been considerable interest in the role that MMPs play in the progression of tumours to metastatic neoplasms. MMPs are upregulated in the vast majority of human and animal tumours and tumour cell lines, ${ }^{27}$ and it is well known that there is a link between MMPs and inflammation and between inflammation and cancer. ${ }^{28}$ Initially, it was believed that MMPs were produced and released by the tumour to degrade the ECM, allowing the tumour to invade nearby tissues; however, it is now apparent that MMPs play a more complex role. A considerable amount of MMP expression in the tumour microenvironment is by stromal cells (as a host response) rather than by the tumour cells directly. ${ }^{29}$ In addition, MMP cleavage of nonmatrix targets results in promotion or inhibition of multiple stages of cancer progression — including 
proliferation, survival, angiogenesis, invasion/ migration and immune evasion. ${ }^{30}$

In neurodegenerative diseases, such as Alzheimer's disease and Parkinson's disease, MMPs have been shown to play important but complex roles. MMPs have been shown to increase bloodbrain barrier permeability that can cause oedema, haemorrhage and cell death and is thought to play a role in white matter damage. ${ }^{31}$ In Alzheimer's disease, ADAMs contribute to the formation of amyloid- $\beta$ peptides, and MMPs (especially MMP9) function in their clearance; drugs that affect both classes are likely to have complex effects. ${ }^{32}$ In Parkinson's disease, MMP3 is released from apoptotic neurones in vitro. MMP3 degrades extracellular macromolecules, but also induces microglia to release TNF, causing further neuronal death. ${ }^{33}$

Although there is a large body of evidence implicating various MMPs in disease pathologies, MMP inhibitors (MMPIs) have proved problematic. A number of clinical trials using MMPIs, especially in cancer, have failed spectacularly, for a number of reasons. $^{29,30}$ Many of the trials failed because of poor efficacy and adverse effects. Often, these were attributed to off-target effects of broad spectrum inhibitors. In many of the disease models listed above, one MMP provides protective effects while another causes disease symptoms; inhibiting both produces a complex spectrum of results, at best. Another reason for failure in cancer trials is poor design. MMPIs are cytostatic and, therefore, using the same measures of efficacy as with cytotoxic compounds will not yield clear results. Another problem with cytostatic treatments is the requirement for early and long-term treatment; side effects are thus unacceptable. Many off-target effects have been attributed to off-target MMP inhibition or inhibition of non-MMP metalloproteinases (especially the ADAMs and ADAM-TSs).

\section{Conclusions}

The MMP family plays important roles in many physiological functions, including ECM remodelling, cell growth/proliferation and inflammation: over- or under-expression has been associated with many disease states. The family consists of 23 genes in humans and 23 genes in mice, which vary in substrate specificity and expression levels in the body. Although clinical trials have failed in the past decade, there is still interest in trying to inhibit specific MMPs, although targeted and careful use is warranted. ${ }^{20}$ Often in the same disease, different MMPs act in disease progression while others act to suppress the disease state; it is therefore important to understand the effects of each MMP that is inhibited and its role in the pathogenesis of the disease.

\section{Acknowledgments}

We thank our colleagues for valuable discussions and a careful reading of this manuscript. This work was supported, in part, by NIH grants R01EY17963 and R21AA017754 (V.V.) and P30ES06096 (D.W.N.).

\section{References}

1. Huxley-Jones, J., Clarke, T.K., Beck, C., Toubaris, G. et al. (2007), 'The evolution of the vertebrate metzincins; insights from Ciona intestinalis and Danio rerio', BMC Evol. Biol. Vol. 7, p. 63.

2. Cauwe, B., Van den Steen, P.E. and Opdenakker, G. (2007), 'The biochemical, biological, and pathological kaleidoscope of cell surface substrates processed by matrix metalloproteinases', Crit. Rev. Biochem. Mol. Biol. Vol. 42, pp. 113-185.

3. Gross, J. and Lapiere, C.M. (1962), 'Collagenolytic activity in amphibian tissues: A tissue culture assay', Proc. Natl. Acad. Sci. USA Vol. 48, pp. 1014-1022.

4. Nagase, H., Barrett, A.J. and Woessner, J.F., Jr. (1992), 'Nomenclature and glossary of the matrix metalloproteinases', Matrix Suppl. Vol. 1, pp. $421-424$.

5. Sternlicht, M.D. and Werb, Z. (2001), 'How matrix metalloproteinases regulate cell behavior', Annu. Rev. Cell Dev. Biol. Vol. 17, pp. 463-516.

6. Visse, R. and Nagase, H. (2003), 'Matrix metalloproteinases and tissue inhibitors of metalloproteinases: Structure, function, and biochemistry', Circ. Res. Vol. 92, pp. 827-839.

7. Le, N.T., Xue, M., Castelnoble, L.A. and Jackson, C.J. (2007), 'The dual personalities of matrix metalloproteinases in inflammation', Front. Biosci. Vol. 12, pp. 1475-1487.

8. Nagase, H., Visse, R. and Murphy, G. (2006), 'Structure and function of matrix metalloproteinases and TIMPs', Cardiovasc. Res. Vol. 69, pp. $562-573$.

9. Van Wart, H.E. and Birkedal-Hansen, H. (1990), 'The cysteine switch: A principle of regulation of metalloproteinase activity with potential applicability to the entire matrix metalloproteinase gene family', Proc. Natl. Acad. Sci. USA Vol. 87, pp. 5578-5582.

10. Piccard, H., Van den Steen, P.E. and Opdenakker, G. (2007), 'Hemopexin domains as multifunctional liganding modules in matrix metalloproteinases and other proteins', J. Leukoc. Biol. Vol. 81, pp. $870-892$

11. Fernandez-Patron, C., Zouki, C., Whittal, R., Cwan, J.S. et al. (2001), 'Matrix metalloproteinases regulate neutrophil-endothelial cell adhesion through generation of endothelin-1[1-32]', FASEB J. Vol. 15, pp. $2230-2240$. 
12. Banda, M.J., Rice, A.G., Griffin, G.L. and Senior, R.M. (1988), 'Alpha 1-proteinase inhibitor is a neutrophil chemoattractant after proteolytic inactivation by macrophage elastase', J. Biol. Chem. Vol. 263, pp. 4481-4484.

13. Gearing, A.J., Beckett, P., Christodoulou, M., Churchill, M. et al. (1994), 'Processing of tumour necrosis factor-alpha precursor by metalloproteinases', Nature Vol. 370, pp. 555-557.

14. Gearing, A.J., Beckett, P., Christodoulou, M., Churchill, M. et al. (1995), 'Matrix metalloproteinases and processing of pro-TNF-alpha', J. Leukoc. Biol. Vol. 57, pp. 774-777.

15. Ito, A., Mukaiyama, A., Itoh, Y., Nagase, H. et al. (1996), 'Degradation of interleukin 1 beta by matrix metalloproteinases', J. Biol. Chem. Vol 271, pp. 14657-14660.

16. Yu, Q. and Stamenkovic, I. (2000), 'Cell surface-localized matrix metalloproteinase-9 proteolytically activates TGF-beta and promotes tumor invasion and angiogenesis', Genes Dev. Vol. 14, pp. 163-176.

17. Schultz, G.S. and Wysocki, A. (2009), 'Interactions between extracellular matrix and growth factors in wound healing', Wound Repair Regen. Vol. 17, pp. $153-162$.

18. Pei, D. and Weiss, S.J. (1995), 'Furin-dependent intracellular activation of the human stromelysin-3 zymogen', Nature Vol. 375, pp. 244-247.

19. Massova, I., Kotra, L.P., Fridman, R. and Mobashery, S. (1998), 'Matrix metalloproteinases: Structures, evolution, and diversification', FASEB J. Vol. 12, pp. 1075-1095

20. Fingleton, B. (2007), 'Matrix metalloproteinases as valid clinical targets', Curr. Pharm. Des. Vol. 13, pp. 333-346.

21. Gruber, B.L., Sorbi, D., French, D.L., Marchese, M.J. et al. (1996), 'Markedly elevated serum MMP-9 (gelatinase B) levels in rheumatoid arthritis: A potentially useful laboratory marker', Clin. Immunol. Immunopathol. Vol. 78, pp. 161-171.

22. Itoh, T., Matsuda, H., Tanioka, M., Kuwabara, K. et al. (2002), 'The role of matrix metalloproteinase-2 and matrix metalloproteinase-9 in antibody-induced arthritis', J. Immunol. Vol. 169, pp. 2643-2647.
23. Toriseva, M. and Kahari, V.M. (2009), 'Proteinases in cutaneous wound healing', Cell Mol. Life Sci. Vol. 66, pp. 203-224.

24. Daheshia, M. (2005), 'Therapeutic inhibition of matrix metalloproteinases for the treatment of chronic obstructive pulmonary disease (COPD)', Curr. Med. Res. Opin. Vol. 21, pp. 587-594.

25. Lagente, V., Manoury, B., Nenan, S., Le Quement, C. et al. (2005), 'Role of matrix metalloproteinases in the development of airway inflammation and remodeling', Braz. J. Med. Biol. Res. Vol. 38, pp. $1521-1530$

26. Hautamaki, R.D., Kobayashi, D.K., Senior, R.M. and Swapiro, S.D. (1997), 'Requirement for macrophage elastase for cigarette smoke-induced emphysema in mice', Science Vol. 277, pp. 2002-2004.

27. McCawley, L.J. and Matrisian, L.M. (2000), 'Matrix metalloproteinases: Multifunctional contributors to tumor progression', Mol. Med. Today Vol. 6, pp. 149-156.

28. Philip, M., Rowley, D.A. and Schreiber, H. (2004), 'Inflammation as a tumor promoter in cancer induction', Semin. Cancer Biol. Vol. 14, pp. $433-439$.

29. Coussens, L.M., Fingleton, B. and Matrisian, L.M. (2002), 'Matrix metalloproteinase inhibitors and cancer: Trials and tribulations', Science Vol. 295, pp. 2387-2392.

30. Pavlaki, M. and Zucker, S. (2003), 'Matrix metalloproteinase inhibitors (MMPIs): The beginning of phase I or the termination of phase III clinical trials', Cancer Metastasis Rev. Vol. 22, pp. 177-203.

31. Rosenberg, G.A. (2009), 'Matrix metalloproteinases and their multiple roles in neurodegenerative diseases', Lancet Neurol. Vol. 8, pp. 205-216.

32. Yin, K.J., Cirrito, J.R., Yan, P., Hu, X. et al. (2006), 'Matrix metalloproteinases expressed by astrocytes mediate extracellular amyloid-beta peptide catabolism', J. Neurosci. Vol. 26, pp. 10939-10948.

33. Choi, D.H., Kim, E.M., Son, H.J., Joh, T.H. et al. (2008), 'A novel intracellular role of matrix metalloproteinase-3 during apoptosis of dopaminergic cells', J. Neurochem. Vol. 106, pp. 405-415. 